\title{
NO $_{x}$ AND HETEROGENEITY EFFECTS IN HIGH LEVEL WASTE (HLW)
}

\author{
PI: Dan Meisel, Notre Dame Radiation Laboratory, and Department of Chemistry and \\ Biochemistry, University of Notre Dame, Notre Dame, IN 46556 \\ PI: Donald M. Camaioni, Environmental and Health Sciences Division, Pacific Northwest \\ National Laboratory, Richland, WA 99352. \\ PI: Thom Orlando, Environmental Molecular Science Laboratory, Pacific Northwest National \\ Laboratory, Richland, WA 99352.
}

We summarize contributions from our EMSP supported research to several fieldoperations of the Office of Environmental Management (EM). In particular we emphasize its impact on safety programs at the Hanford and other EM sites where storage, maintenance and handling of HLW is a major mission. In recent years we were engaged in coordinated efforts to understand the chemistry initiated by radiation in HLW. Three projects of the EMSP ("The $\mathrm{NO}_{x}$ System in Nuclear Waste," "Mechanisms and Kinetics of Organic Aging in High Level Nuclear Wastes, D. Camaioni - PI" and "Interfacial Radiolysis Effects in Tanks Waste, T. Orlando - PI") were involved in that effort, which included a team at Argonne, later moved to the University of Notre Dame, and two teams at the Pacific Northwest National Laboratory. Much effort was invested in integrating the results of the scientific studies into the engineering operations via coordination meetings and participation in various stages of the resolution of some of the outstanding safety issues at the sites. However, in this Abstract we summarize the effort at Notre Dame.

Two broad issues were identified as bottlenecks in understanding the chemistry in HLW a) the heterogeneity of the systems and b) the relatively poorly understood chemistry of NOx species (rather than water degradation products). As a result of the EMSP projects mentioned above the following advancement were achieved, starting with the NOx issue:

1. All of the initial water primary products are converted to the $\mathrm{NO} 2$ or $\mathrm{NO}$ oxidizing species. The rates of these conversions were quantified and novel routes for the conversions outlined. Redox potentials of the relevant species were measured. 2. The hypothesis that NO2 is the major oxidant of organic compounds in the HLW was confirmed. Relative rates, and in some cases absolute rates, of the NO2 radical with organic substrates (mostly chelates and their degradation products) were measured. Mechanisms and rates of the reaction of relevant organic radicals with $\mathrm{NO} 2$, and $\mathrm{NO} 2-$ were collected. 3. These quantitative measurements were incorporated into a simplified computer model that describes the radiation-induced chemistry of HLW simulants and their mixtures.

On the heterogeneity issue, the following discoveries can be noted:

1. All electrons that are produced by the radiation in solid (silica) particles, at least up to $22 \mathrm{~nm}$ in diameter, escape the solid and appear in the water. Thus small particles (or high porosity and large surface area materials) will not prevent some of the effects of radiation on water. 2. All the holes that are generated in the same solid are trapped in the particles. Oxidative processes may or may not (depending on the trap in the solid) be prevented by the solid particle. 3. Either one of these observations can be manipulated by modifying the surface of the particles. However, merely changing the surface charge will not alter the initial conclusions. 4 . The charge separation that occurs across the solid/water interface, as described above, may lead to back- 
reactions at later stages of the radiolytic effects. Thus, $\mathrm{H} 2$ yields may be modified because of reoxidation of molecular hydrogen by trapped holes.

Specific technological problems that utilized the scientific knowledge developed here can now be outlined: 1. Quantitative computation of the rate of the degradation of organic compounds in HLW simulants was instrumental in the resolution of the organic tanks issue. 2. The outlined mechanisms, and later quantitative calculation of the rate of hazardous gas generation in simulants, eventually lead to predictive calculations of gas generation in waste tanks. 3. Estimates of the rate of gas generation at the surface of wet solid fissile materials, stored for transportation in sealed canisters, is becoming more realistic. 